\title{
A FRAMEWORK FOR OPTIMAL QUALITY of PARTS IN REVERSE ENGineERING
}

\author{
Nebojša Rašović \& Milenko Obad
}
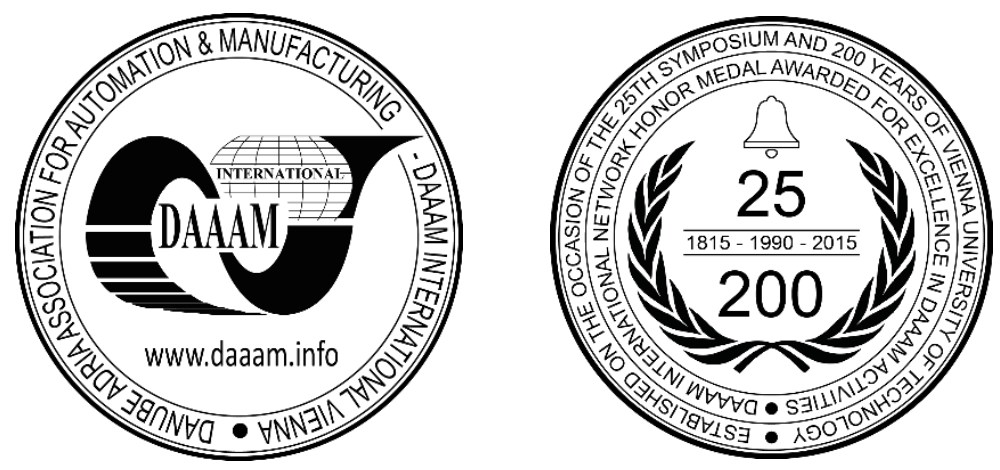

This Publication has to be referred as: Rasovic, N[ebojsa] \& Obad, M[ilenko] (2016). A Framework for Optimal Quality of Parts in Reverse Engineering, Proceedings of the 27th DAAAM International Symposium, pp.0328-0337, B. Katalinic (Ed.), Published by DAAAM International, ISBN 978-3-902734-08-2, ISSN 1726-9679, Vienna, Austria DOI: $10.2507 / 27$ th.daaam.proceedings.048

\begin{abstract}
This paper presents a methodology of reverse engineering process in order to obtain an optimal quality of the scanned surfaces. These surfaces are using for model reconstruction in process which analyses curved geometry. This kind of design process has a key role in modern engineering, because the goal is to get electronic input, in case where a physic model is missing from some reason or is not available. By corresponding software support, we are able to control surfaces and their vector definition. Using advanced techniques, it is possible to set a procedure for measuring complex parts dimensions and transforming them on behalf of design intent. For the paper purpose, it is used physic model of the screw to create the point cloud. Therefore, the crucial phase of reverse engineering process is developing of adequate method for the best manufacturing conditions. Case study has been carried out by NextEngine 3D Scanner ULTRA HD.
\end{abstract}

Keywords: Reverse engineering; Feature recognition; Surface recognition.

\section{Introduction}

Today, product development has a tendency for rapidly positioning at the marketplace by various ways. One of these ways for product development is certainly Computer Aided Reverse Engineering (CARE). The CARE process has a few steps before getting a final result, such as usable electronic input. First of all, it is necessary to make measurements at points on the part surfaces. These points are defined by own $\mathrm{x}, \mathrm{y}, \mathrm{z}$ values of the space coordinate system. The CARE system forms collection of these points named point cloud. Point structure helps in feature construction with respect to the components or solids geometry. This approach allows multiple inspections of the real components or of the existing CAD models. For visualization inspection, geometric model has to be discretized into a mesh composition of a finite number of primitive elements [1]. High quality inspection is a real challenge in all activities of modern engineering. Some researchers are proposing structured methodology for the screening and comparison based on programs that are suitable for quality inspection [2].

Reverse engineering is modern technology which provides a lot of benefits and possibilities in the CAD/CAM domain [3]. There are a large number of reasons for using reverse engineering. Some of them are including design reconstruction with impacts on shape analysis and prototyping [4, 5], quality control and inspection on certain parts [6], request for making clone of the original model [7], remanufacture of existing parts [8], and more. Very important fact about reverse engineering process is based on effort to eliminate inaccuracy in the proposed scan algorithm [9]. The generic process 
has three main phases. The first one is scanning selected part, second one belongs to the point cloud processing in certain 3D software modeler, and the least one is application on specific area of geometric model development (Fig. 1).

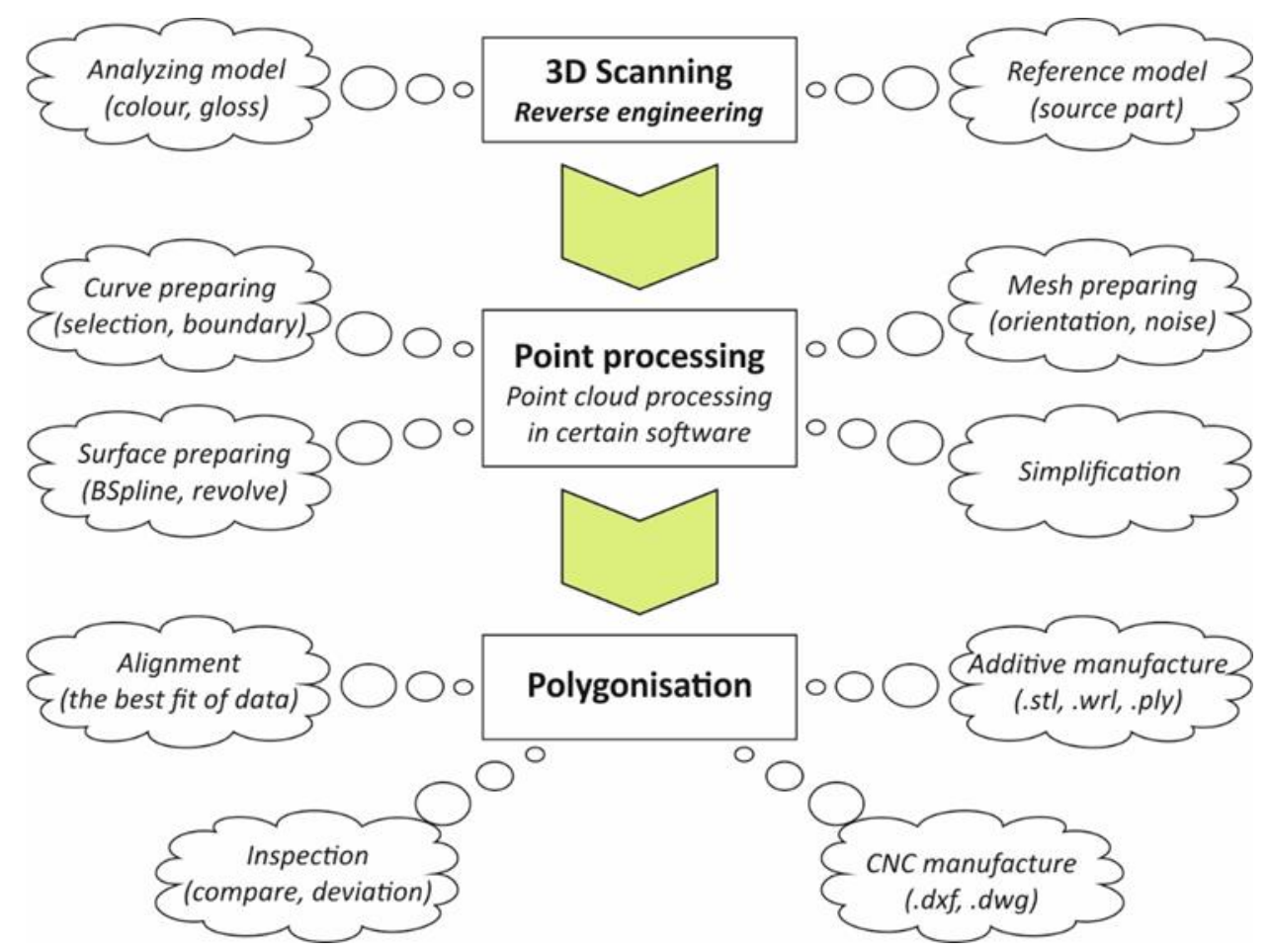

Fig. 1. Generic Algorithm of reverse engineering process

The initial phase involves preparatory activities. First of all, it performs analyses of model appearance in order to solve light fracture issue. This statement occurs at glossy models, where the light is reflected from the surface. According to the above mentioned, it must be adopted a strategy for selected adequate parameters which are available in scanner system options.

The second phase is important from a model of geometric standpoint. Scan data are using for presentation of the point cloud data. The point cloud data sets of data points in a coordinate system, where the points are defined by $\mathrm{x}, \mathrm{y}, \mathrm{z}$ coordinates, which are a surface of some object. 3D scanner creates the point cloud and measures a large number of points of a surface. The results of this phase are introducing into the next phase of reverse engineering process (Fig. 2).

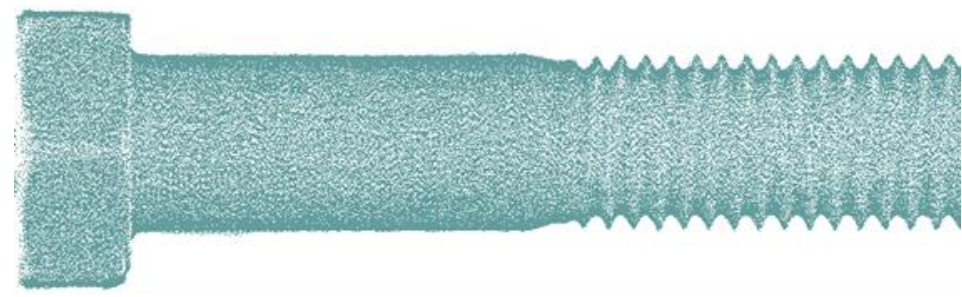

Fig. 2. A point cloud of the screw

The third phase includes a set of activities which provide different direction of application. The point clouds are converted to a polygon or a triangle mesh, or CAD formats through a process referred as a surface reconstruction. The point cloud of a model can be aligned to a CAD model or even another point cloud and compared to check for deviations. These deviations are displayed such as colour maps that give a visual overview of the deviation between the manufactured part and the CAD model. Geometric dimensions and tolerances can also be extracted directly from the point cloud. Getting a usable CAD model presents the hardest thing in the scanning process. The output of this step is a final product of the model geometry written by one of the standard formats in CAD/CAM systems such as IGES, STEP, STL, VRML, PLY, DXF, DEG etc.

\section{3D Scanning process}

For the paper purpose, scan data and following processes are carried out by NextEngine 3D Scanner ULTRA HD, which represents a high resolution device with quickly captures millions of points. The equipment consists of scanner, 
multidrive, part gripper and platter (Fig. 3). In this way, there is no need for calculating distance in order to have cable connection between scanner and multidrive.

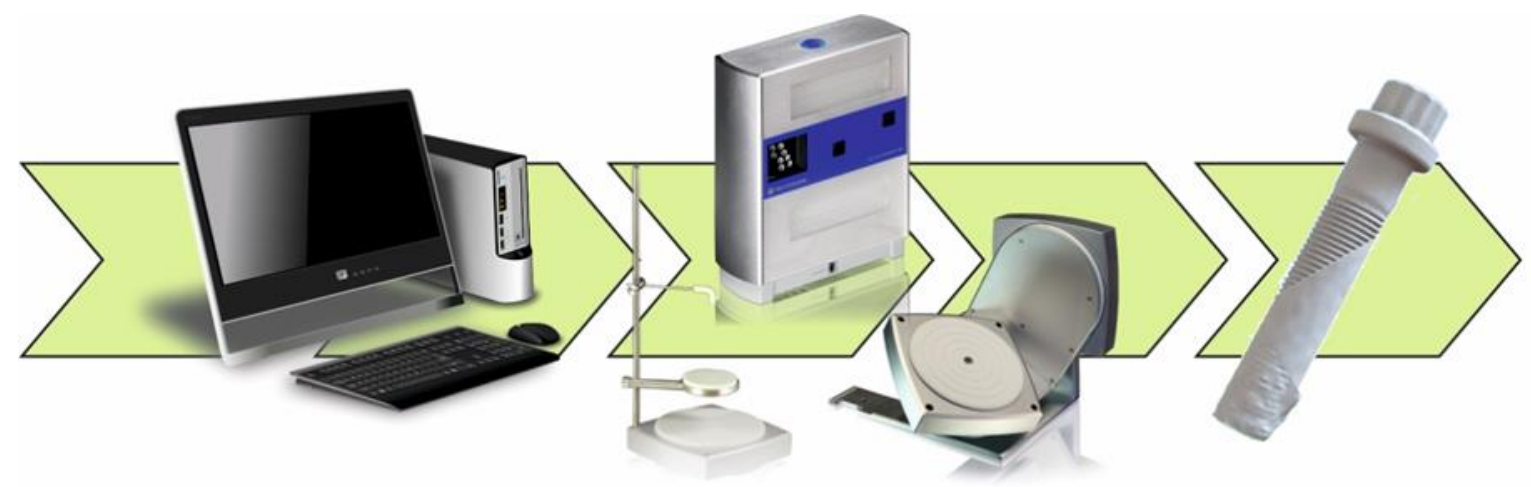

Fig. 3. Apparatuses of the scanning process

Scanning process is carried out in ScanStudio v2.0.2 NextEngine Inc. Before the first scanning, there is a possibility for calibration process of multidrive in order to align a model for well capture reasons. This step is highly recommended, but it is always possible to jump for another step. Models have to be analysed from skin point of view since the laser beam may be reflected in undesired directions from shiny or glossy surfaces. According to that, there is an opportunity for powder coating. For this case of the screw, model has grey matt surfaces all over the volume, so that gives a well capture results of pictures. In the scan options, a selection is made by both, the HD Speed for the greatest resolution and the highest colour accuracy under texture capture mode. An user can control predefines parameters of regenerating points from raw scan data. This applies a simplification of the model before starting of the process, which helps to remove unwanted areas and to decrease complications.

All above mentioned is an important framework condition at the beginning of the scan process. Except that, it is possible to manipulate with five families for scan positioning related to the divisions, start angle, tilt angle, points per inch square, and target. All of them are predefined parameters, but it is always possible for manual setup. After the scan process, the scan data are presented on the Fig. 4.

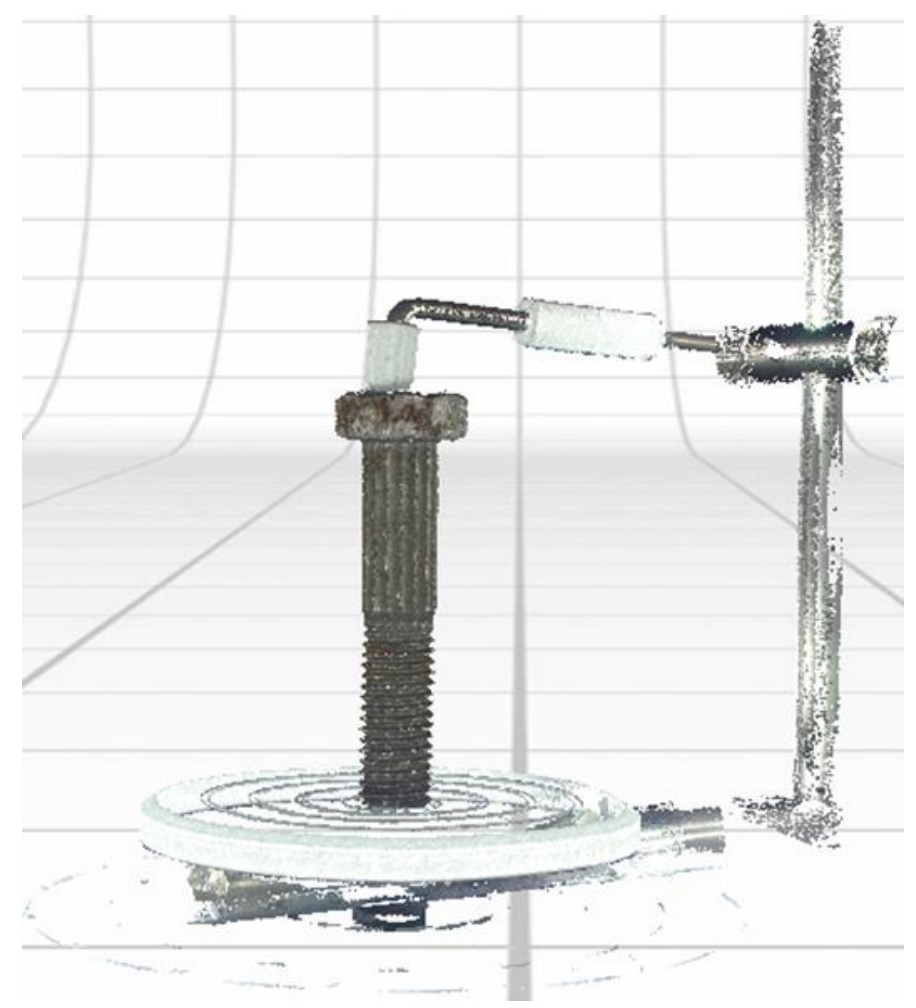

Fig. 4. Raw scan data of the screw

The conditions have been created for applying the most popular common tools, which are typical for the CAD environment. It is obvious, that a part gripper and a platter are over plus and need to be removed by trim tool. Overlapping data have to be trimmed in order to improve fusing, which is the next step in this process (Fig. 5). The selected areas have 
been marked by red colour, and those are overlap data. This is useful in direction of getting smaller file data and it becomes easily for handling. It is always calculated to reduce file data in order to disburden computer hardware for better results in data processing.

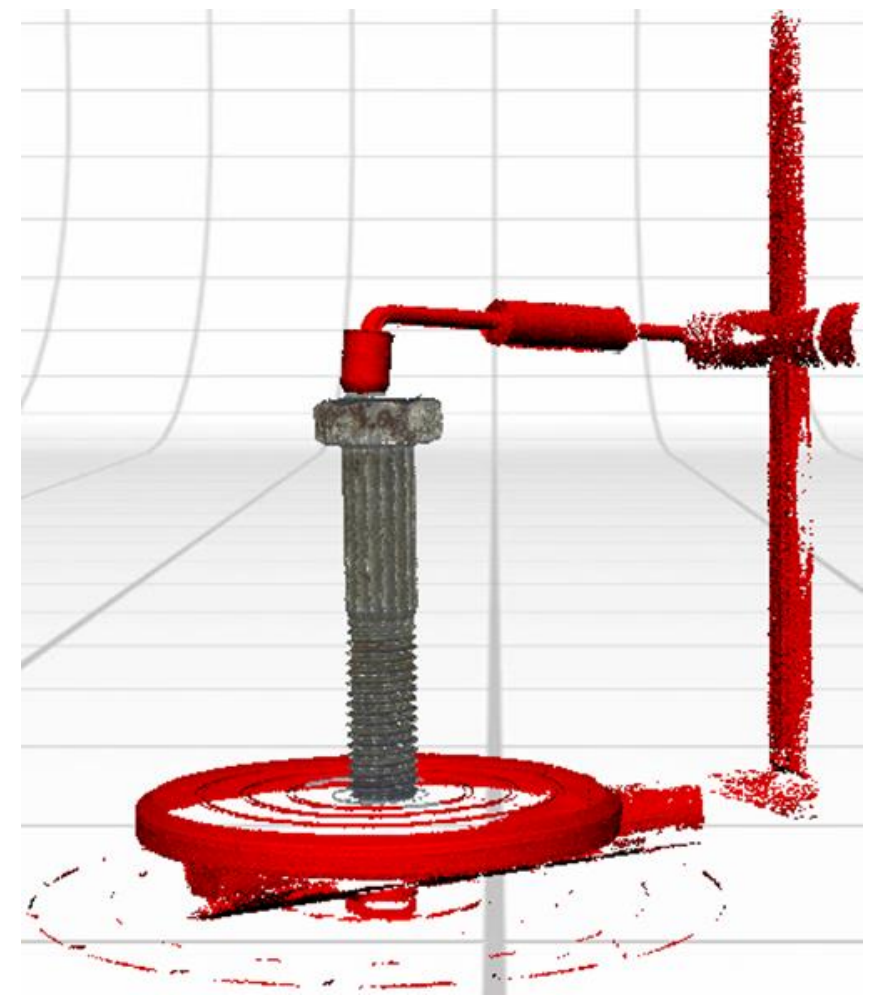

Fig. 5. Highlighted surfaces have to be trimmed

The fuse tool is intended for merges, re-meshes, holes fill, and simplifies scan data. It would be desirable to achieve mesh simplification by entering a deviation tolerance. That makes the file size smaller. It is smart simplification, which means that keeps more points in detailed areas and less points in other areas. Also, the model needs to be polished to get smoothness surfaces. It is possible to polish entire model or some areas, especially holes or threads. In that case, a selector must add a surface for starting process of filling (Fig. 6). The entire model has to be buffed.
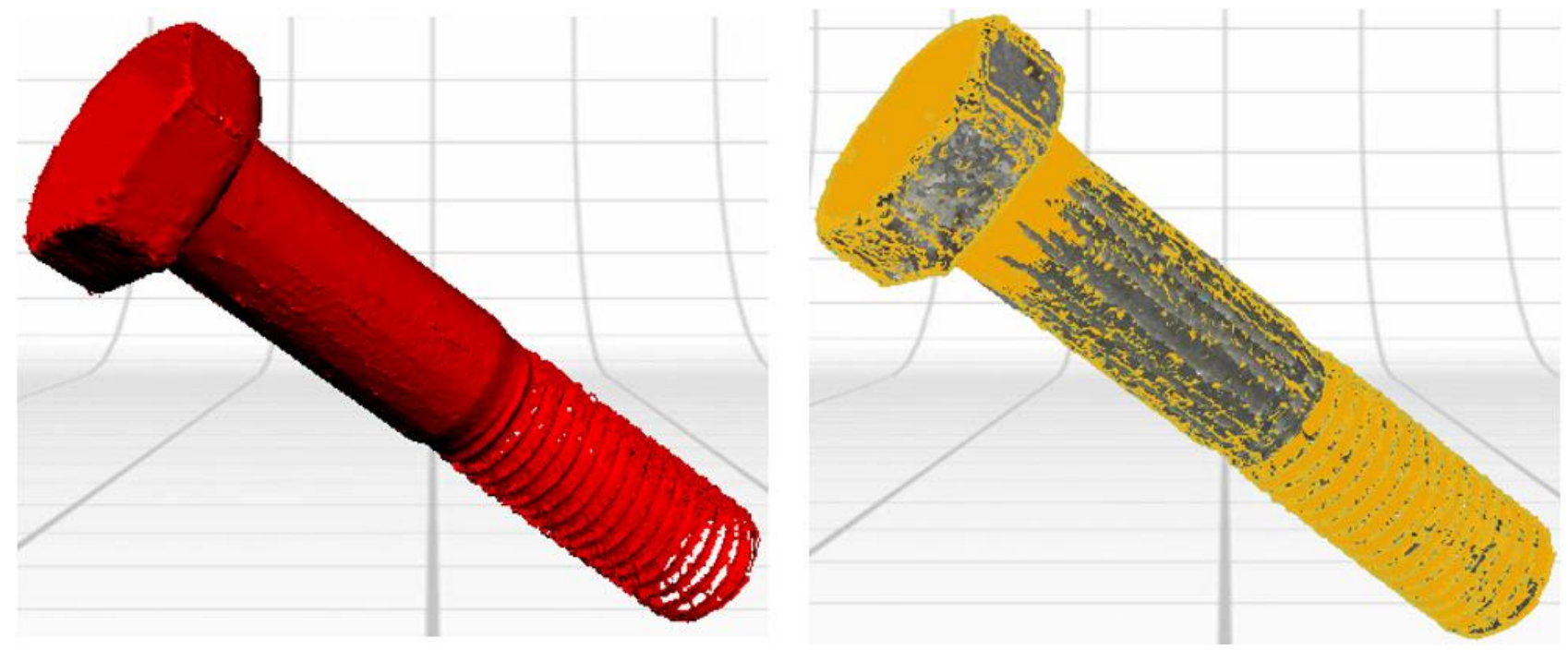

Fig. 6. Filling model areas using available fill tools by software

\section{Point cloud processing}

After forming a point cloud data, it has been created conditions for importing geometry in SolidWorks, by Dassault Systemes. First of all, file data are importing such as .xyz extension. An imported point cloud looks like a dense mesh with a lot of numbers of active points (Fig. 7). 


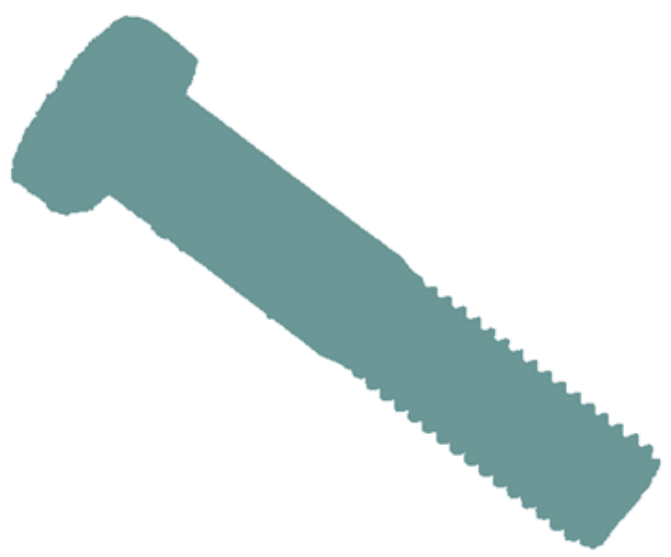

Fig. 7. A point cloud imported in SolidWorks

A point cloud needs to be analyzed over mesh preparation wizard. On the start phase, a file data has 268353 points. This value has to be much smaller. A point cloud orientation uses the existing orientation for the mesh obtained by importing. A lot of errors are always introduced into scan data where the points are placed in unwanted areas or regions. For the point cloud data, noise removal tool divides the cloud into partitions and removes points that are outside the average distribution, resulting in the removal of noise points. It is necessary to remove separate mesh patches that are small in an area. As a possibility, wizard is offered to delete extraneous point data, but it has already done in ScanStudio v2.0.2 NextEngine, Inc. Simplification reduces the number of vertices for mesh features, or reduces the number of points for point cloud features, resulting in a simpler, smaller file size in additional. Now, we simplify the entire mesh. The percentage value for reduction is about $70 \%$, and resulting by 71155 target points size (Fig. 8).

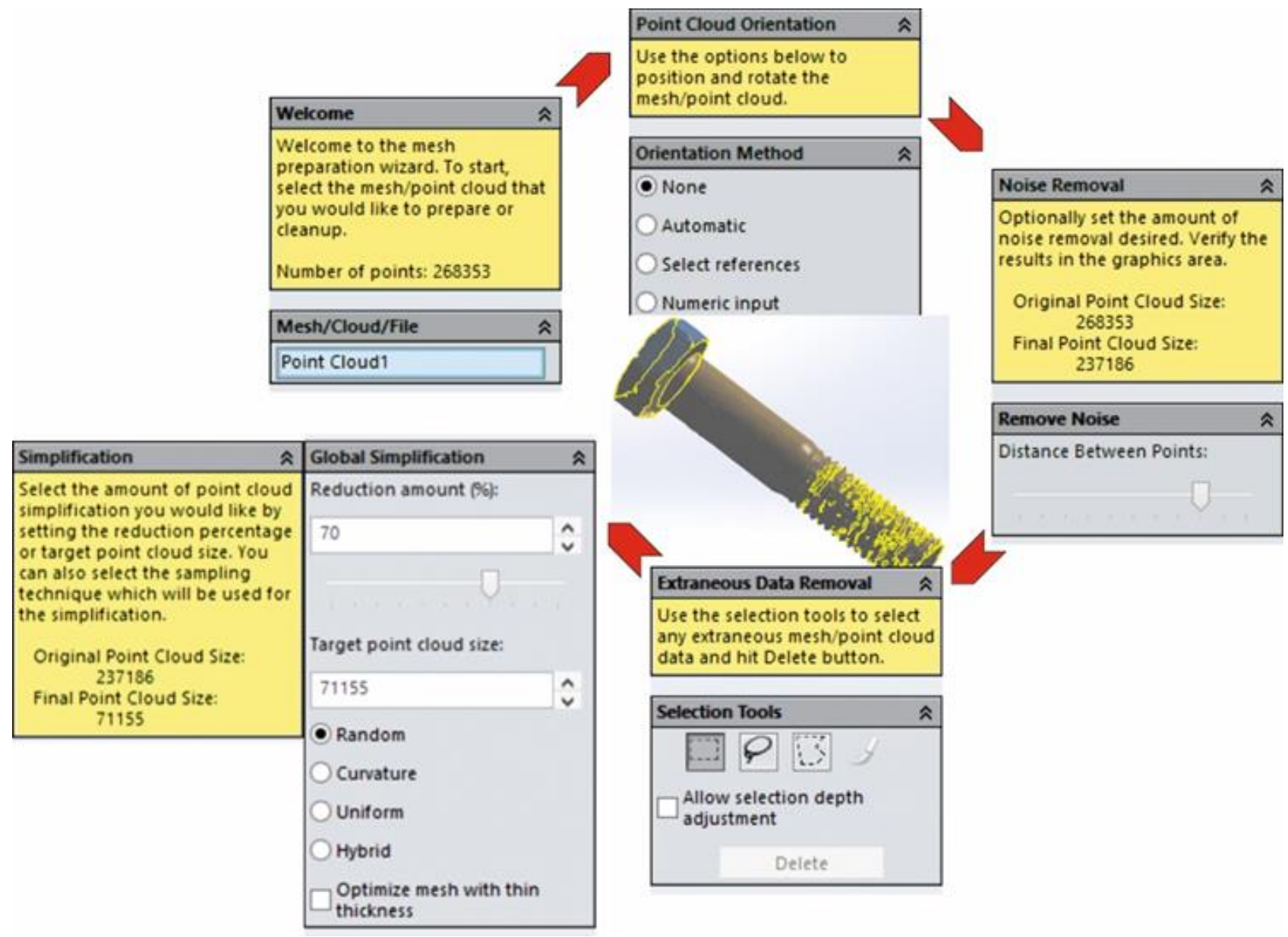

Fig. 8. Mesh preparation process 
Level of smoothness can be adjusted by global smoothing slider to set the overall value and for individual regions as well (Fig. 9, left). Also, all holes are automatically detected to be filled. You can control which holes need to be removed, or filled up (Fig. 9, right).
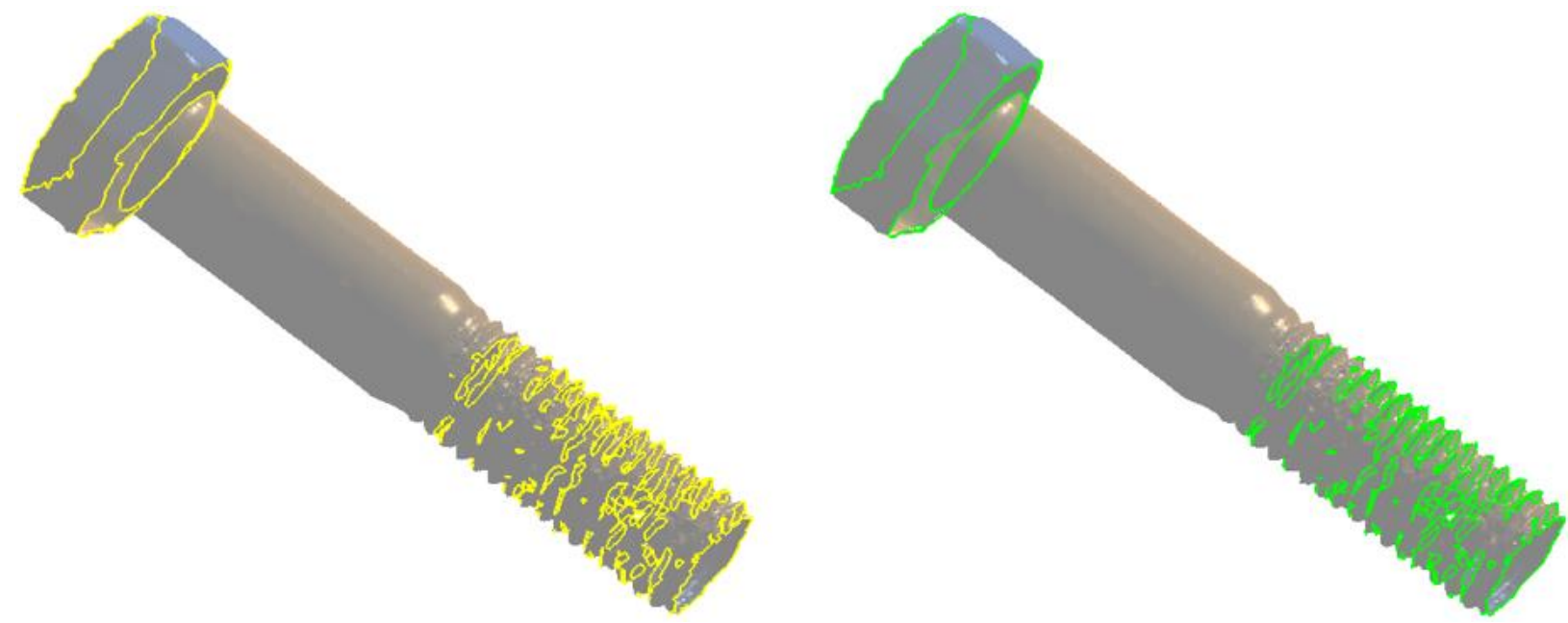

Fig. 9. Process of surfaces smoothing (left) and filling holes (right)

Upon getting the mesh, it is necessary to analyze accuracy of the curved geometry. A curve wizard offers to create boundary and section curves in point cloud and mesh files. The key of this methodology is the fact that the curves are created in 3D sketch and are fully editable. That's obvious, it can be controlled the surface accuracy by parametric curve. Also, an user can add points for more level of control. In this case, section plane is top plane, and will serve for creating set of parallel reference offset planes on the certain distance. Before that, it has to select a pick point on the model, to set active point from that place for distance measuring. For this model, number of faces for setting up the parameters and editing of the curve's points and selection have to be reduced at the minimum. A start point belongs to the top plane of the model, and that place is at the head screw. Total number of the reference planes are 19 and distance between them are $3.73288117 \mathrm{~mm}$ (Fig. 10).

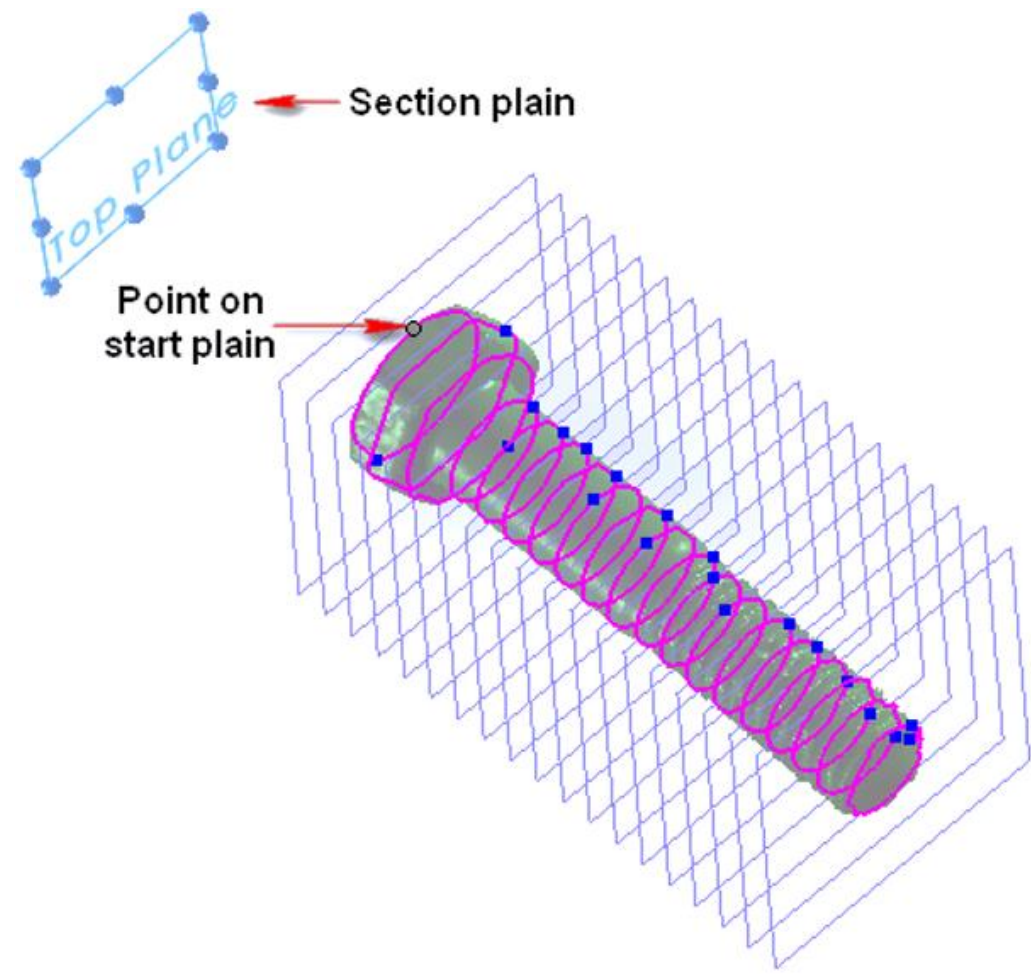

\begin{tabular}{|c|c|c|}
\hline Mes! & /Cloud/File & ᄎ \\
\hline Mes & & \\
\hline Creat & ion Method & ᄎ \\
\hline OD & screte & \\
\hline (C) St & ction & \\
\hline $\mathrm{OB}$ & undary & \\
\hline Secti & on Plane Parameters & ᄎ \\
\hline$\pi_{k}$ & Top Plane & \\
\hline 10 & Point@Mesh & \\
\hline$\hat{K}_{D}^{\lambda}$ & $3.73288117 \mathrm{~mm}$ & $\hat{v}$ \\
\hline $0^{\circ} \#$ & 19 & $\hat{v}$ \\
\hline
\end{tabular}

Fig. 10. Adding reference planes parallel with the top plane 
Each curve is a sketch in certain plane and contains editable points for accuracy control. In the parametric form, each point on a curve is expressed as a function of a parameter $t$ by equations: $x=x(t), y=y(t), z=z(t)$. Equations in this form are parametric equations for $\mathrm{x}, \mathrm{y}$, and $\mathrm{z}$. Equation (1) is the parametric equation for a $3 \mathrm{D}$ curve:

$$
L(t)=[x, y, z]^{T}=[x(t), y(t), z(t)]^{T} ; \text { where is: } t_{\min }<t<t_{\max }
$$

First of all, it should be selected a curve in editing curve circumstances, and should be adjusted or modified the curve's shape using edit tools such as a traditional CAD user interface. In the other word, if a curve is selected from the offered list, then there is a situation to adjust the curve fit.

A curve fitting should be adjusted by controlling value of the tolerance. If the slider is on the left side of scale, sample more vertices or points, making the curve fit more tightly to the mesh or moves it to the right to loosen the curve fit. If it adds more points, that situation refines the curve position. You can create a curve which more accurately represents the designer's intent by removing points or vertices related to noise in the scanned data (Fig. 11).
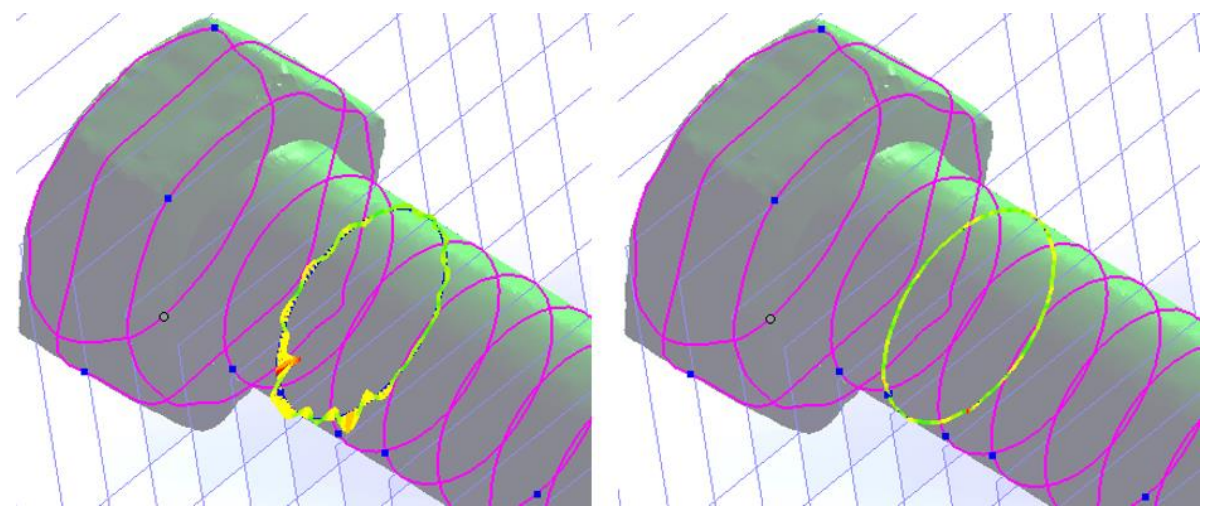

Fig. 11. Adjusting curve fit

In order to control all surfaces and convert them to the solid body, it is necessary to running surfaces wizard analyzed on the existing mesh. Accordance with previous, you need select individual regions in the graphic area or automatically extract all offered faces which are recognized by 3D modeler (Fig. 12).
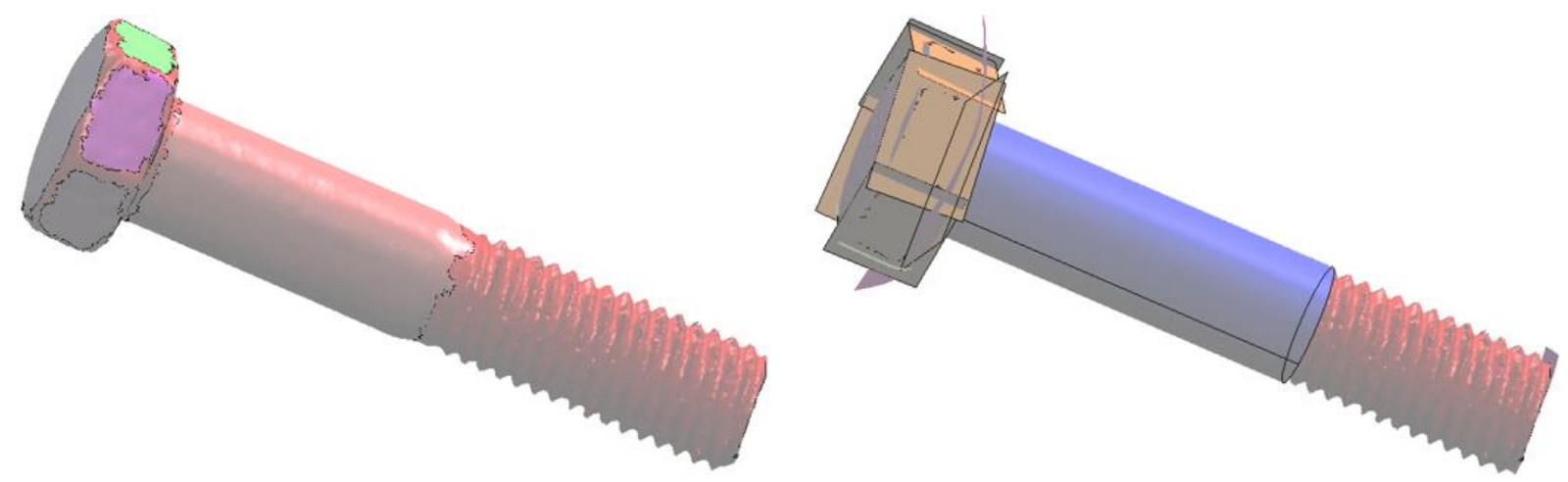

Fig. 12. Extracting a surfaces

A surface wizard helps in simplification of individual surface. It recognizes error on some surfaces and offers to remove them from configuration. Also, it is possible to relax some geometric entities in order to get accurate object, which is a crucial phase in next phase called inspection with original CAD model. After job realisation, the wizard highlighting with a message that the surface model is completed and recommended to inspect the model. A final model layout looks like as shown on the Fig. 13. 


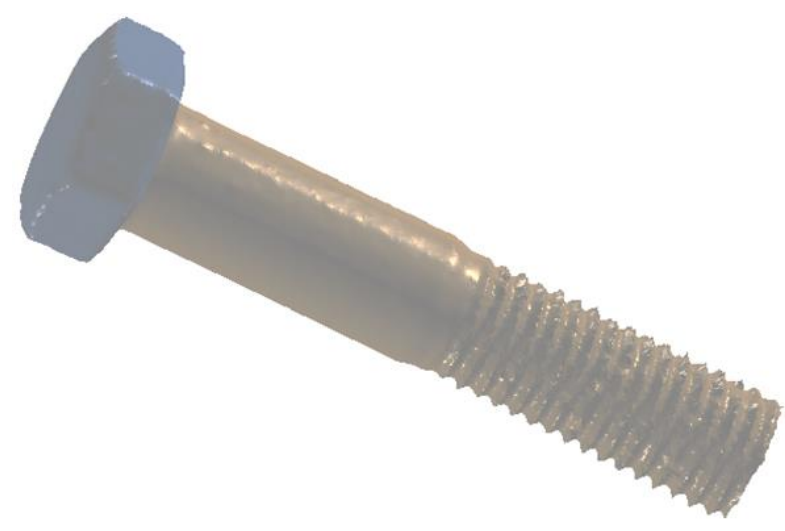

Fig. 13. Final look of the model

\section{Polygonisation}

A surface's shape consists of the set of faces. These faces involve a series of geometric entities which has played a key role in forming single entity. Surface quality is important factor in approach of the products design process [10, 11]. The ability to provide the geometry from its original and making better transition from vectors formats to physical model are major challenge in engineering. In order to verify produced shape and its fit and function within assembly, it is implemented the measuring technique by 3D scanner. Scanner is supplied by geometric data from the existing physical object. Thus obtained geometric data are used by accuracy analyse of the object surfaces on standard screw. For the best results, all measurements are automatically merged into one to achieve a common global coordinate system. To perform a comparison of scanned physical and CAD source data, the scanned data are converted into CAD data coordinate position. For comparison purposes, the system requires from user to define the nominal point first at the CAD model, but then to select actual point at the scanned model and repeat that three times. These align operations have been realized in GOM Inspect software. Based on defined reference points, the system calculates the best fit for the actual coordinates and suggests a possible alignment (Fig. 14).
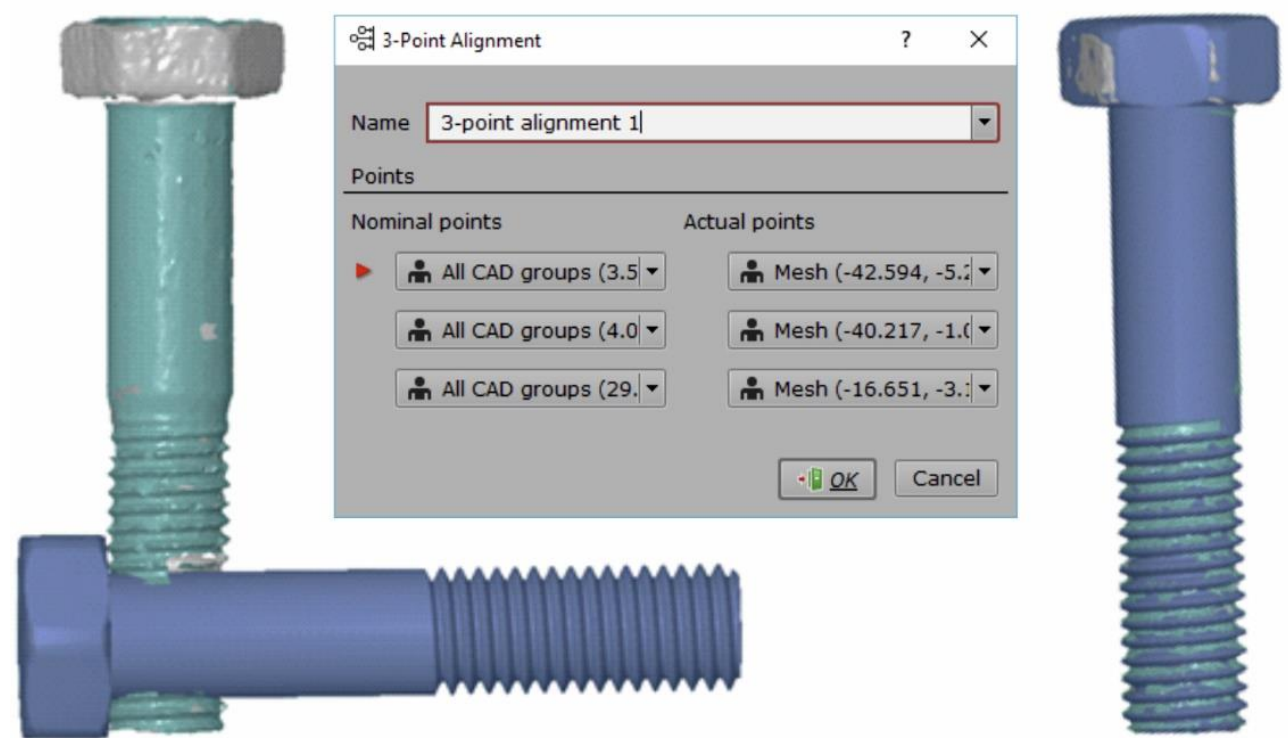

Fig. 14. Models fitting by 3-Point Alignment

In order to visualize surfaces of the models, colors are added to each particular part of the objects. It has been created a color map for easier interpretation and transferring dimensional precision of the produced model, which are compared by the CAD model. The process has done by deviation inspection. It automatically displays colorful deviation scale, which is based on the precisely calculated deviation values. Reddish areas on the map indicate that the scanned surfaces of the test pieces are over measured dimensions in relate to the surfaces of the CAD model. In contrast, the blue areas on the map indicate that the scanned surfaces of the test pieces are measured according to the surfaces of the CAD model. The green areas are the closest to the real dimensions, while the rest of the model is coincident with real dimensions. Deviation scale can also be finely adjusted or adapted by the user (Fig. 15). 


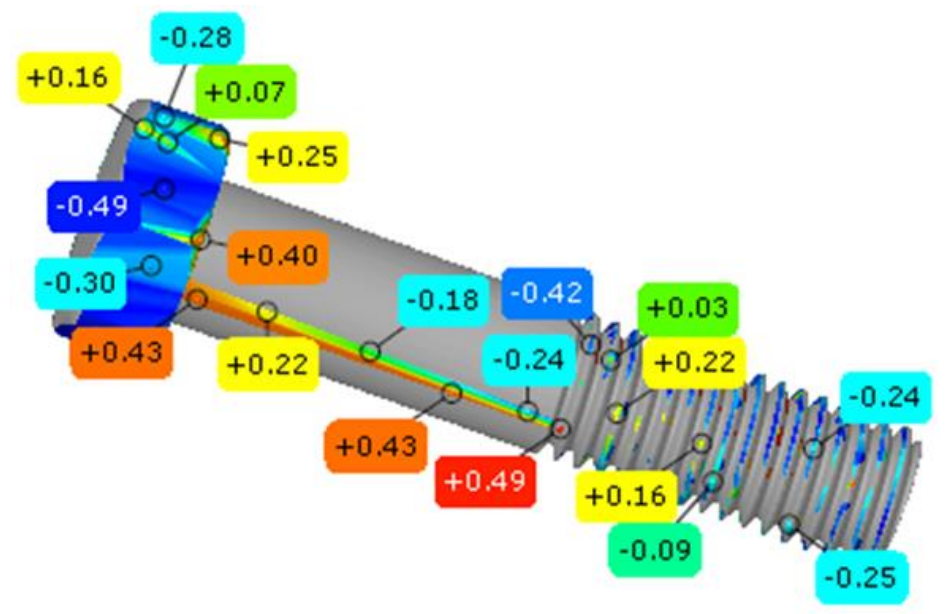

Fig. 15. A Surfaces deviation of the scanned data compared by the CAD model

Based on comparing approach and deviation inspection, it can see clear differences in surfaces precision. The real condition is that the comparing model is approximation of the original part. The main objective has been improved the quality and precision of the polygon model of the screw. On the first view, it is obviously clear that deviation is in the range from $+0.5 \mathrm{~mm}$ to $-0.5 \mathrm{~mm}$, because the colour map highlights the extremely red colour, which gives a clear picture of the deviation of the part. Cylindrical face is recognized in 3D modeller and confirmed like that. In accordance with that, cylindrical face has zero deviation value on the over $90 \%$ of the body (Fig. 15).

The reason that attracts attention is certainly thread part of the volume. From the other hand, thread has more complicated geometry and deviation level is varying. Thread will be more accurate if reference planes have been smaller distance between each other. In that case, there is a solution for creating various distance along the entire body. One distance value for the cylindrical face and another one distance value for the thread. This situation shows a real state of the thread because configuration need to be uniform for better fit. That case brings a smaller number of faces and contributions to model simplification. This note of uniform and variable model configuration will be one of the subjects of the further researches in this area. The extension of this file is prepared for manufacturing by the certain method, additive or CNC.

\section{Conclusion}

In order to define the appropriate form, designers use various methods of formulation of the product. In any case, the final result is a form, which is precisely defined in terms of proportions and surface classification. While the proportions being relatively easily identifiable and defined, the definition of areas are great challenge of the whole process. In the most cases, designers still produce physical prototypes from design clay. The prototypes are then digitized using a 3D scanner. The result of the scan is a point cloud in space over which surfaces are laid using various techniques.

This paper has presented the framework for optimal quality of parts in reverse engineering process. Based on that, a solution is obtained for acquiring scanned data and their post-processing in some procedures. The overview of the scan process has included parameters which define conditions for better point cloud approximation. The research goal was to coincidence all measurements at actual model compared by CAD model. Polygonisation showed certain amount of surfaces deviation in some segments of the screw. But on the other side, it is getting fully usable 3D model for potential production by additive manufacturing methods.

The goal of the future work is to define an advanced framework of mathematical characteristics of model surfaces, which can be used to describe parametric surfaces. This process shall be conditioned by the skills and experience of an engineer who performs parameterization. The end surfaces definitions are, in spite of the mathematical notation, usually complex. Production processes are not limited any more to conventional technology. New layered manufacturing technologies enforce rapidly. Regarding to the manufacturing process, design recommendations for the design of products will be improved in the future.

\section{References}

[1] Bénière, R., Subsol, G., Gesquière, G., Le Breton, F. \& Puech, W., (2013) A comprehensive process of reverse engineering from 3D meshes to CAD models. Computer-Aided Design. 45(11): p. 1382-1393

[2] Minetola, P., Iuliano, L. \& Calignano, F., (2015) A customer oriented methodology for reverse engineering software selection in the computer aided inspection scenario. Computers in Industry. 67: p. 54-71 
[3] Vinesh Raja \& Fernandes, K.J., (2008) Reverse Engineering, Springer London

[4] Wang, J., Gu, D., Yu, Z., Tan, C. \& Zhou, L., (2012) A framework for 3D model reconstruction in reverse engineering. Computers \& Industrial Engineering. 63(4): p. 1189-1200

[5] Min, Z., (2011) A New Approach of Composite Surface Reconstruction Based on Reverse Engineering. Procedia Engineering. 23: p. 594-599

[6] Marciniec, A., Budzik, G., Dziubek, T. \& Grzelka, M.a., (2012) Quality control and inspection of bevel gears of the aircraft gearbox utilizing the Atos 3d Scanner. Journal of KONES. Powertrain and Transport Journal of KONES. Powertrain and Transport. 19(1): p. 261-266

[7] Jiang, Q., Feng, X., Gong, Y., Song, L., Ran, S. \& Cui, J., (2016) Reverse modelling of natural rock joints using 3D scanning and 3D printing. Computers and Geotechnics. 73: p. 210-220

[8] Paulic, M., Irgolic, T., Balic, J., Cus, F., Cupar, A., Brajlih, T. \& Drstvensek, I., (2014) Reverse Engineering of Parts with Optical Scanning and Additive Manufacturing. Procedia Engineering. 69: p. 795-803

[9] Kovács, I., Várady, T. \& Salvi, P., (2015) Applying geometric constraints for perfecting CAD models in reverse engineering. Graphical Models. 82: p. 44-57

[10] Cekic, A., Rasovic, N., Obad, M., Kaljun, J., Dolsak, B. \& Begic-Hajdarevic, D., (2016) Production of Optimized Layered Products using Intelligent Support. Proceedings of the 26th DAAAM International Symposium, DAAAM International, Vienna, Austria, pp. 0271-0279. DOI: 10.2507/26th.daaam.proceedings.037

[11] Rašović, N., (2014) Intelligent decision support in design process of products to be produced by layered manufacturing technologies. Ph.D. Thesis, University of Mostar: Mostar, BiH 